Scientific Visualization, 2021, volume 13, number 3, pages 34 - 46, DOI: 10.26583/sv.13.3.04

\title{
Analysis and Visualization of the Computational Experiments Results on the Comparative Assessment of OpenFOAM Solvers Accuracy for a Rarefaction Wave Problem
}

\author{
A.E. Bondarev ${ }^{1}$, A.E. Kuvshinnikov² \\ Keldysh Institute of Applied Mathematics RAS \\ 1 ORCID: oooo-0003-3681-5212, bond@keldysh.ru \\ 2 ORCID: 0000-0003-1667-6307, kuvsh9o@yandex.ru
}

\begin{abstract}
The article is devoted to the analysis and visualization of the generalized computational experiment results on the comparative analysis of the accuracy for a group of open software package OpenFOAM solvers. The basic problem here is the classical problem of the rarefaction wave formation in a flow. This problem has an exact solution which we use as etalon. The generalized computational experiment makes it possible to carry out parametric studies in a grid partition of the region in the space of defining parameters. The construction of a generalized computational experiment is based on the synthesis of mathematical modeling, parallel technologies and visual analytics. As a rule, the results obtained in such an experiment are multidimensional data, on the basis of which the objective functional is constructed, which is the main object of interest of the researcher. In this case, the defining parameters are the input stream Mach number and the flow deflection angle. The deviation from the exact solution calculated in the L1 and L2 norms is considered in the role of the objective functional. For each solver, a parametric problem is solved by varying the Mach number and flow deflection angle. The results obtained represent the dependence of the objective functional on the defining parameters for each solver. The results are presented in visual form as surfaces and in analytical form as polynomials.
\end{abstract}

Keywords: comparative assessment of accuracy, OpenFOAM solvers, generalized computational experiment, rarefaction wave, processing of visual results, analytical form.

\section{Introduction}

The need to organize and conduct a comparative assessment of the numerical methods accuracy is growing significantly today. The reason for this is the emergence of a large number of new numerical methods, solvers developed on their basis, integrated into various software packages. At the same time, solver developers are far from always able to organize full testing and comparative assessment of accuracy on problems that have a reference solution. It should be remembered that, ultimately, both software packages and the solvers implemented in them are targeted at the end user who uses these software tools to solve practical problems. In this case, the end user must rely on something, and here comparative estimates of the solvers accuracy can help him. In order to provide the most comprehensive and complete comparison, it is necessary to carry out parametric studies. They allow comparative assessment not for one specific problem, but for a class of problems. Parametric numerical research is now being carried out very actively in various fields [1-4].

A generalized computational experiment will help to organize such numerical studies. The theory and methods of generalized computational experiment are being actively developed at the Keldysh Institute of Applied Mathematics of the RAS. A generalized computational experiment is a computational technology based on the synthesis of solutions to problems of mathematical modeling, the use of parallel technologies and methods of visual 
analytics. The construction of such an experiment makes it possible to obtain a numerical solution for a class of problems determined by variations in the defining parameters in certain ranges. The region of the defining parameters is subdivided into a grid. At each point of such a partition, the problem of mathematical modeling is solved using parallel technologies. The result of such a calculation is multidimensional data, with the help of which valuable functionals are constructed as functions of several variables. The obtained data and functionals are explored using scientific visualization and visual analytics methods. The theoretical description of methods, tools and examples of constructing a generalized computational experiment is described in detail in the cycle of works [5-11]. With regard to the tasks of comparative assessment of the accuracy of solvers, the purpose of the study is to obtain comparison results in a visual form and in an analytical form.

This work considers an example of the application of the generalized computational experiment to the problem of accuracy comparative estimation for several solvers of the open software package OpenFOAM [12]. The classical problem of a rarefaction wave formation in a flow is used as the basic problem. The class of problems is determined by the variation in the given ranges of two defining parameters - the freestream Mach number $\mathrm{M}$ and the flow deflection angle $\beta$. This problem has an exact solution described in [13-15]. This solution is used as a reference one. The deviation from the exact solution calculated in the L1 and L2 norms is considered in the role of the objective functional. For each solver, a parametric problem is solved by varying the Mach number and flow deflection angle. The results obtained represent the dependence of the objective functional on the defining parameters for each solver. The results are presented in visual form as surfaces and in analytical form as polynomials. The results obtained make it possible to obtain a fairly complete idea of the accuracy of each of the solvers participating in the comparison in such an important class of problems as the rarefaction wave.

\section{Background - Previous Works}

This work continues a large cycle of works devoted to the development of methods for constructing a generalized computational experiment and its application to practical problems in computational gas dynamics [5-11,17-22]. In particular, the application of a generalized computational experiment to problems of comparative estimation of numerical methods accuracy is considered using examples of typical classes of problems.

A number of characteristic directions presented in previous works can be distinguished:

- the basic principles and methods of constructing a generalized computational experiment for problems of computational gas dynamics are presented in [5-10];

- a description of the main tasks of data visualization arising in the construction of a generalized computational experiment is presented in $[11,16]$;

- a description of the construction and implementation of a generalized computational experiment for problems of comparative assessment of numerical methods accuracy is presented in [17-21]. Papers [17-19] consider the problem of comparing the accuracy of various OpenFOAM solvers when flowing around cones at an angle of attack. This task has four defining parameters. Variations of the Mach number, the cone half-angle, the angle of attack, and the choice of the solver are considered. Results in a similar setting for the problem of of an oblique shock wave formation are presented in [20, 21];

- a description of the construction of analytical dependencies for visual images is presented in [22].

All the approaches, methods and software tools developed at the previous stage are used in this work for a comparative analysis of the OpenFOAM solvers accuracy for the problem of a rarefaction wave formation. 


\section{Description of the test problem}

This work is devoted to the accuracy comparative assessment of OpenFOAM solvers when calculating the rarefaction wave. The comparison of accuracy was carried out on the basis of constructing a generalized computational experiment. The well-known classical problem of two-dimensional rarefaction wave formation in an inviscid gas flow around a flat plate at an angle of attack was considered as the basic problem. A scheme of such a flow is shown in Figure 1. A supersonic flow of an inviscid compressible gas runs onto a flat halfplate at an angle of attack $\beta$, as shown in the figure. A rarefaction wave fan is formed at the end of the plate.

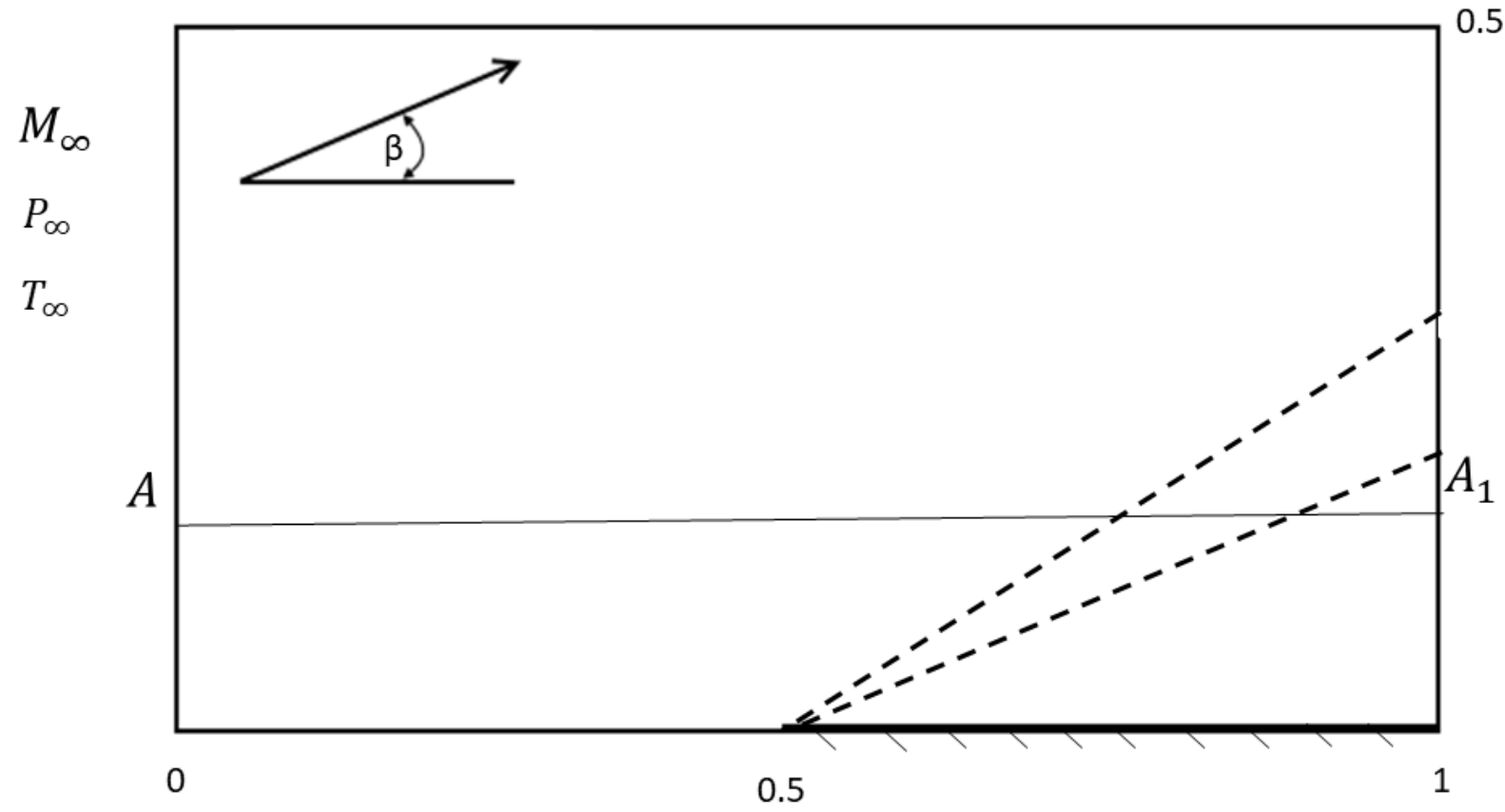

Fig. 1. Flow scheme. The area of calculations.

This problem is widely known as the Prandtl-Mayer flow. This problem has an exact solution, the description of which can be found in [13-15]. The exact solution in our case acts as a standard one.

The problem is solved within the framework of a two-dimensional system of Euler equations for an inviscid gas. At the inlet boundary, the parameters of the unperturbed free stream are set at the Mach number $M$ and a certain value of $\beta$. On the part of the lower boundary corresponding to the flat plate, the no-slip condition is set. At the outlet boundary, the boundary conditions are set for the zero derivatives of the gas-dynamic functions along the normal to the boundary. At the upper boundary for the velocity components, the boundary conditions are set similarly to the conditions for the input boundary. For the rest of the gas dynamic functions of the upper boundary, the conditions are set similarly to the conditions for the outlet boundary.

Parametric studies were carried out for a Mach number equal to 2-4, with a step of 0.5, and an angle $\beta=6^{\circ}, 10^{\circ}, 15^{\circ}, 20^{\circ}$. Four solvers of the OpenFOAM open software package [2429] - rhoCentralFoam, pisoCentralFoam, sonicFoam, and QGDFoam - took part in the comparative assessment of accuracy. For all these solvers, some actions were taken to unify 
the calculations when setting the boundary and initial conditions, similar to the procedures described in [17-21].

\section{OpenFOAM solvers}

This section contains a short description of the solvers of the open source software package OpenFOAM, participating in the study on comparative assessment of their accuracy. The following solvers were used in the calculations:

1) rhoCentralFoam (rCF) - based on the central-upwind scheme, which is a combination of the central-difference and upwind schemes [23]. The original Kurganov-Tadmor scheme [24] is a central difference scheme based on the Lax-Friedrichs scheme. It is characteristic of Godunov-type schemes that the calculation of the flow through the lateral faces of the cell requires the solution of the Riemann problem on the decay of an arbitrary discontinuity. The schemes with a central difference calculate the required values at the next time step without solving the Riemann problem; they integrate according to the Riemann fan. Consequently, there is no need to search for the Riemann integral and expansion of the solution in terms of characteristics, thus methods of this type are less computationally expensive. However, the OpenFOAM software package uses the Kurganov - Noel - Petrova scheme [25], which is a central upstream scheme. The scheme is central, since it does not require the solution of the Riemann problem, and upstream, since the information taken against the flow rate is used to estimate the width of the Riemann fan. This more accurate estimate makes the schemes less dissipative than the original Kurganov-Tadmor scheme.

2) sonicFoam (sF) - based on the PISO (Pressure Implicit with Splitting of Operator) algorithm [26]. The essence of splitting methods is to separate the influence of various terms on the change in momentum in the computational cell. The contribution of the pressure gradient is decoupled from the contribution of spatial transport and viscous terms. When implementing the method, the predicted and corrected values of the velocity and pressure of the flow are introduced. Then the equations for pressure correction and momentum balance are solved iteratively.

3) pisoCentralFoam [27] is a hybrid method, which is a combination of a central-uupwind scheme with the PISO algorithm. To smoothly switch between the implicit version of the Kurganov-Tadmor method and the splitting method, depending on the local flow parameters, a linear mixing of the flow expressions from both methods is used. This solver is not included in the standard set of solvers in the OpenFOAM software package. It was created by an independent team of developers at the Ivannikov Institute for System Programming of the RAS.

4) The QGDFoam solver [28, 29] is based on the system of quasi-gasdynamic equations [30-32], developed by a group of researchers led by B.N. Chetverushkin. A significant difference between the QGD approach and the Navier-Stokes theory is the procedure of space-time averaging to determine the main gas-dynamic quantities. Additional smoothing in time is the reason for the appearance of additional dissipative terms in the QGD system. These dissipative terms have the form of the second spatial derivatives and are proportional to a small parameter having the dimension of time. The presence of a controllable parameter with dissipative terms makes it possible to successfully suppress unwanted oscillations in the numerical simulation of problems with discontinuities. Additional terms appear to be efficient regularizers. This solver is also not included in the standard set of solvers in the OpenFOAM software package. It was developed at Keldysh Institute of Applied Mathematics of the RAS under the leadership of professor T.G. Elizarova.

\section{Analysis and visualization of results}

This section presents the results of the calculations. Figure 2 shows the pressure field in the computational domain for the exact solution calculated in accordance with [13-15]. Figure 3 shows a typical pressure field for all solvers. The figure shows the pressure field calculated 
using the QGDFoam solver. Note that all calculations for the QGDFoam solver were carried out at the value of the parameter $\alpha$, which controls the dissipative properties of the solver, equal to $\alpha=0.1$. The figures show that the calculated pressure field presents a more blurry picture compared to the pressure field for an exact solution.

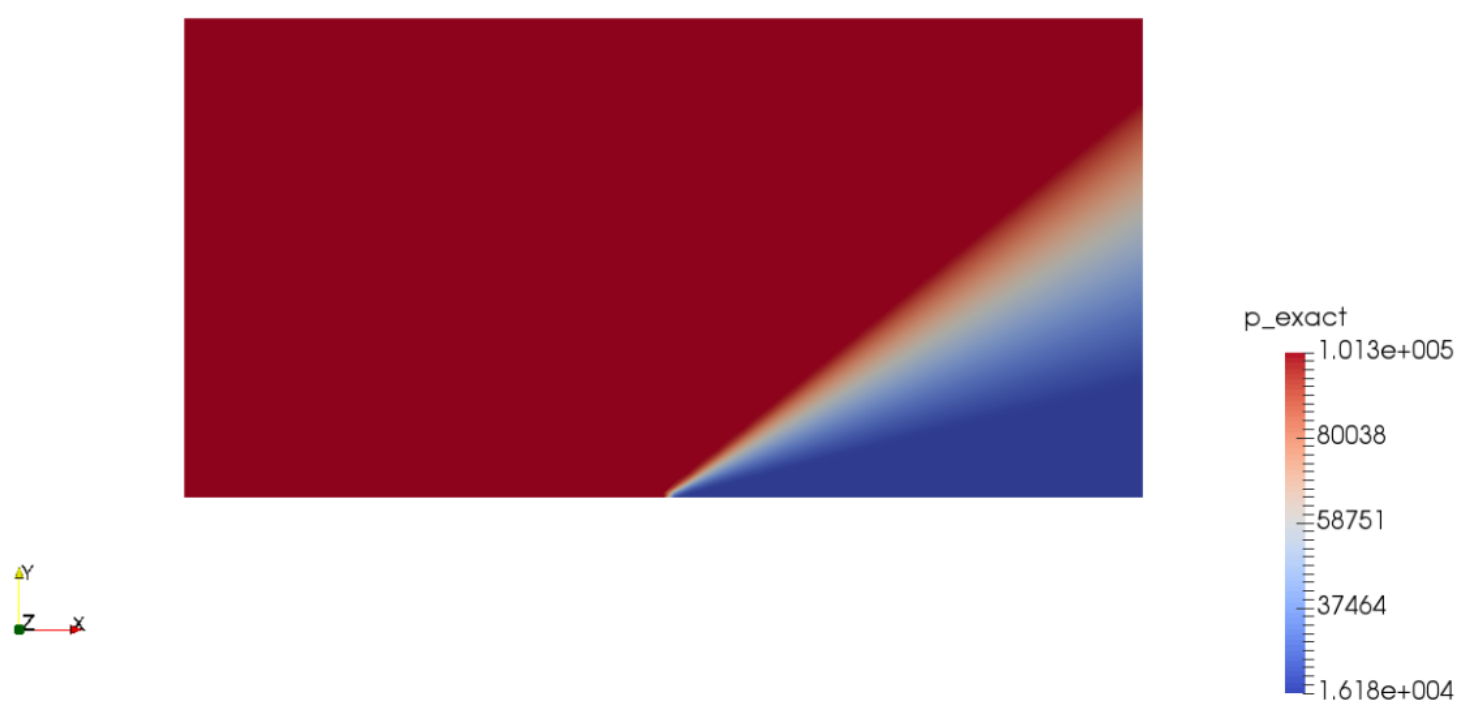

Fig. 2. Pressure field for an exact solution.

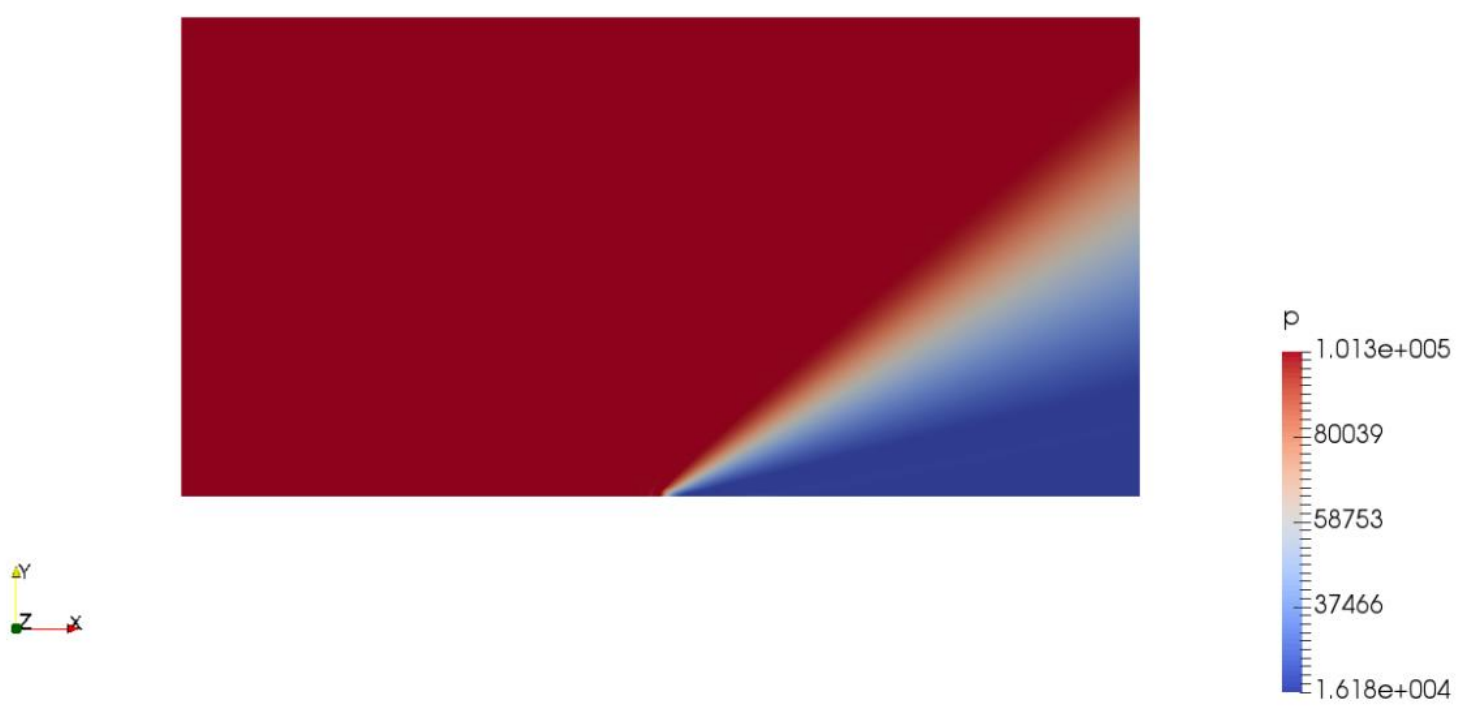

Fig. 3. Pressure field calculated using the QGDFoam solver.

Figure 4 below shows a comparison of the pressure calculated for all solvers with the exact solution along the horizontal line AA1 (Fig. 1). The horizontal line is located at a height of 0.1 from the plate. Figure 5 shows the same comparison in close-up. 


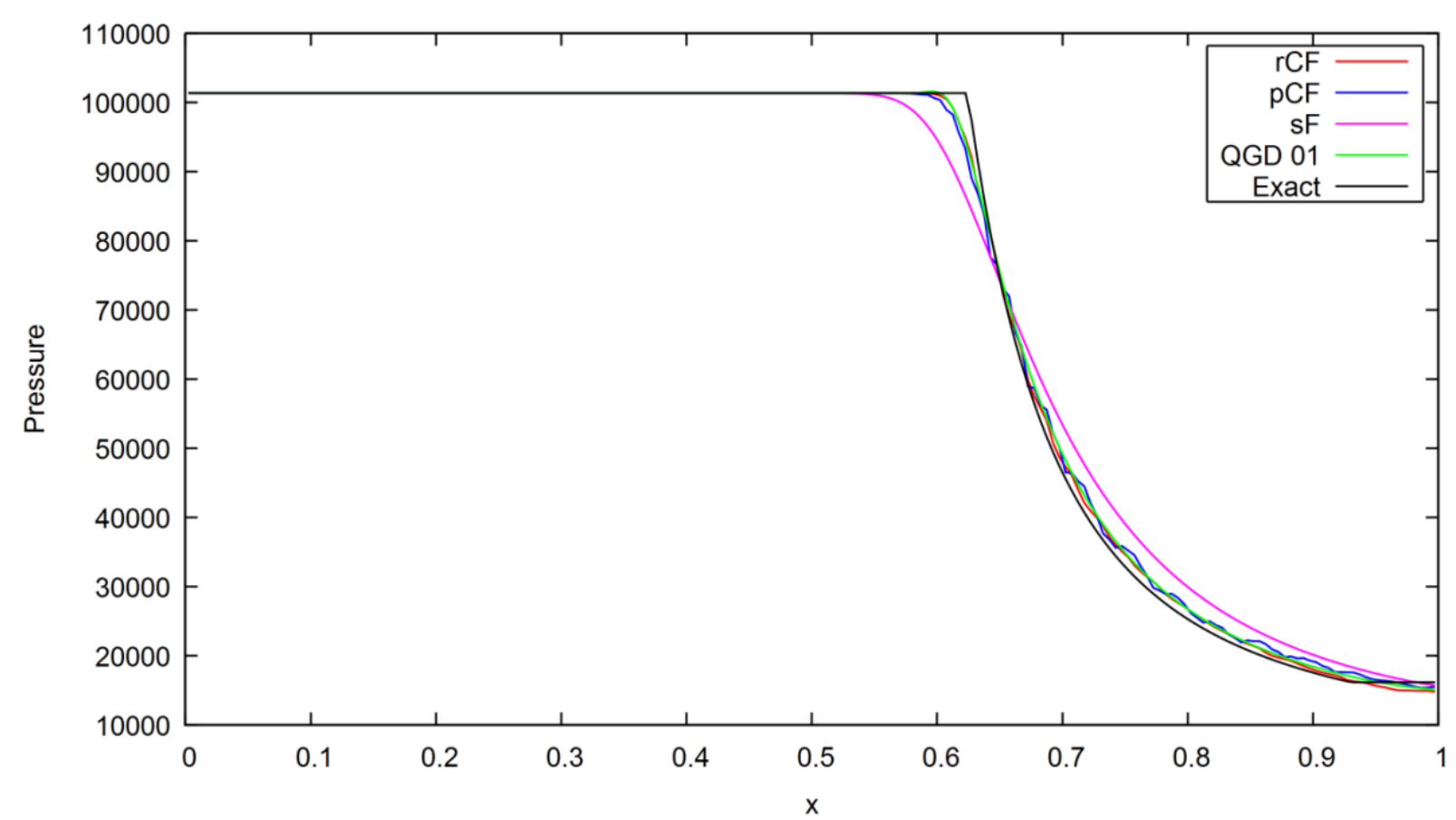

Fig. 4. Comparison of solutions along the horizontal line AA1 for all solvers with exact solution

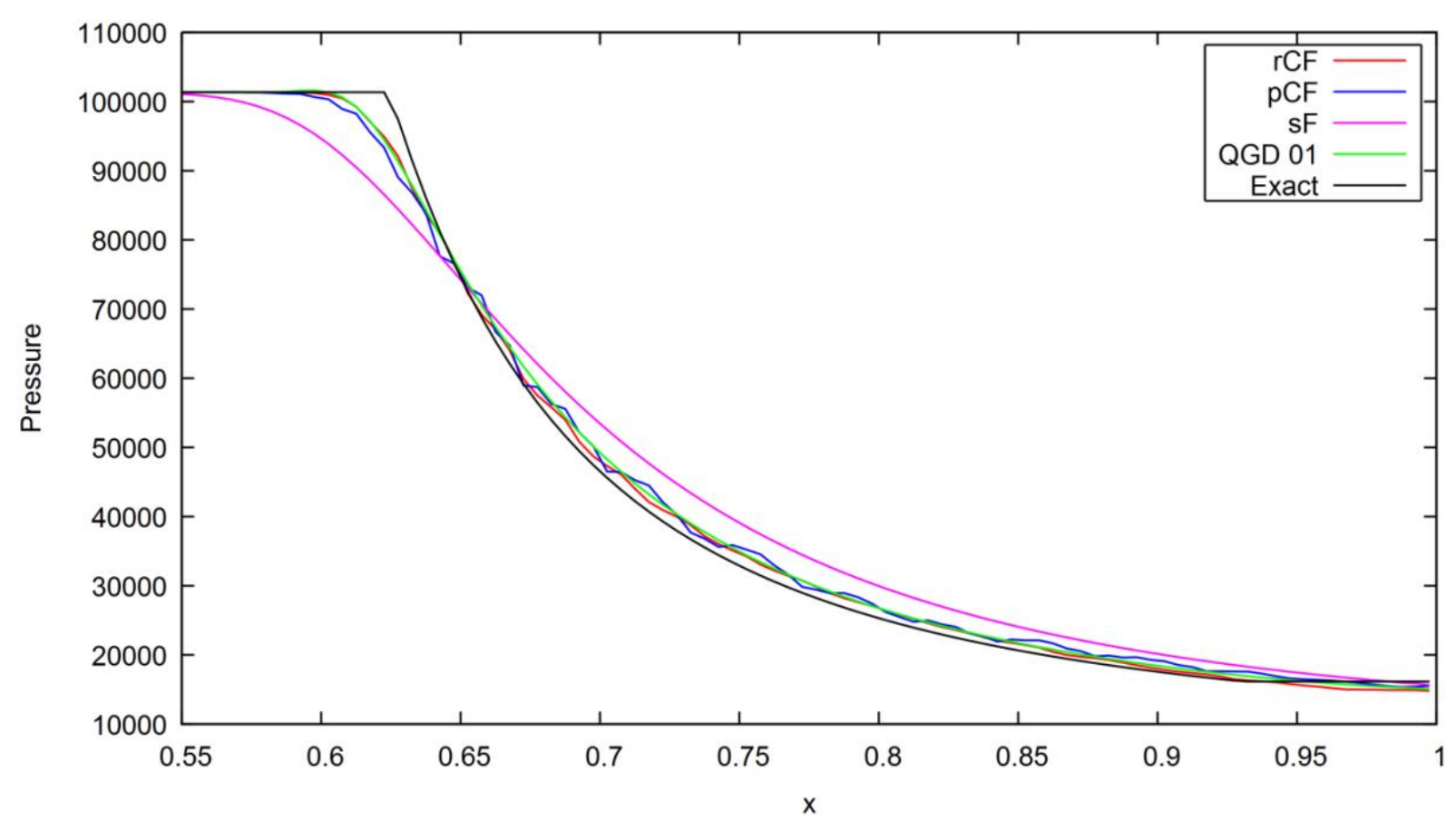

Fig. 5. Comparison of solutions along the horizontal line AA1 for all solvers with the exact solution - close-up.

Figures 4 and 5 show that the sonicFoam solver deviates most from the exact solution. The rhoCentralFoam, pisoCentralFoam and QGDFoam solvers show very close results. It should be noted that the curve for pisoCentralFoam shows constant noticeable fluctuations, which indirectly indicates the presence of unwanted non-destructive oscillations in the solution.

Thus, we have a comparative assessment of the accuracy of the solvers along the line. However, this is clearly not enough. Let us construct estimates of the deviation from the exact solution for the entire computational domain in the L1 and L2 norms. To do this, calculate the relative error Err for the L1 and L2 standards as follows: 


$$
\begin{gathered}
L_{1}: \quad \operatorname{Err}=\sum_{m}\left|y_{m}-y_{m}^{\text {exact }}\right| S_{m} / \sum_{m}\left|y_{m}^{\text {exact }}\right| S_{m} \\
L_{2}: \quad E r r=\sqrt{\sum_{m}\left|y_{m}-y_{m}^{\text {exact }}\right|{ }^{2} S_{m}} / \sqrt{\sum_{m}\left|y_{m}^{\text {exact }}\right|^{2} S_{m}}
\end{gathered}
$$

Here $y_{m}$ is the pressure $p, S_{m}$ is the cell area. The $y_{m}^{\text {exact }}$ values are obtained from the exact solution of the problem of the rarefaction wave formation [13-15]. The parameters of the incident flow vary as follows: the angle of flow deflection $\beta=6^{\circ}, 10^{\circ}, 15^{\circ}, 20^{\circ}$, Mach number $\mathrm{M}=2,2.5,3,3.5,4$. Next, a generalized computational experiment is implemented, based on the results of which we calculate the error for of each solver for each combination $(\mathrm{M}, \beta)$. This enables us to construct for both norms the error in the form of a function of two variables Err $(\mathrm{X} 1, \mathrm{Y} 1)$, where $\mathrm{X} 1=\mathrm{M}$ and $\mathrm{Y} 1=\beta$. These designations will be used in all subsequent figures.

Next, consider a typical picture resulting from the construction of the error Err (X1, Y1) in different norms. For example, consider the results for the QGDFoam solver.

Figure 6 shows the deviation from the exact solution in the L1 norm for the QGDFoam solver in the ranges of the governing parameters. Similarly, Figure 7 shows the results for the L2 norm. By the appearance of the surfaces shown in these figures, it can be argued that the error, in comparison with the exact solution, increases both with an increase in the Mach number and with an increase in the angle of flow deflection.

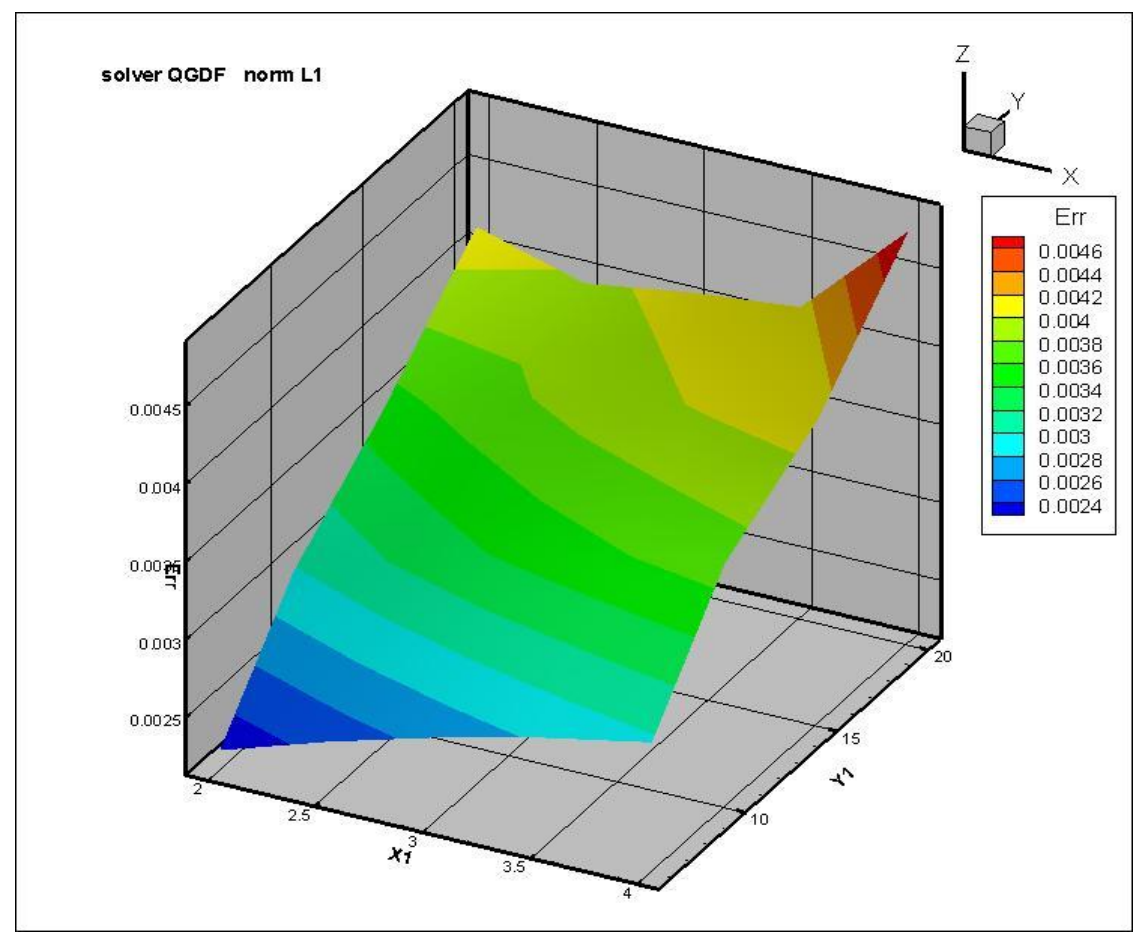

Fig. 6. The deviation from the exact solution in the L1 norm for the QGDFoam solver in the ranges of variation of the governing parameters 


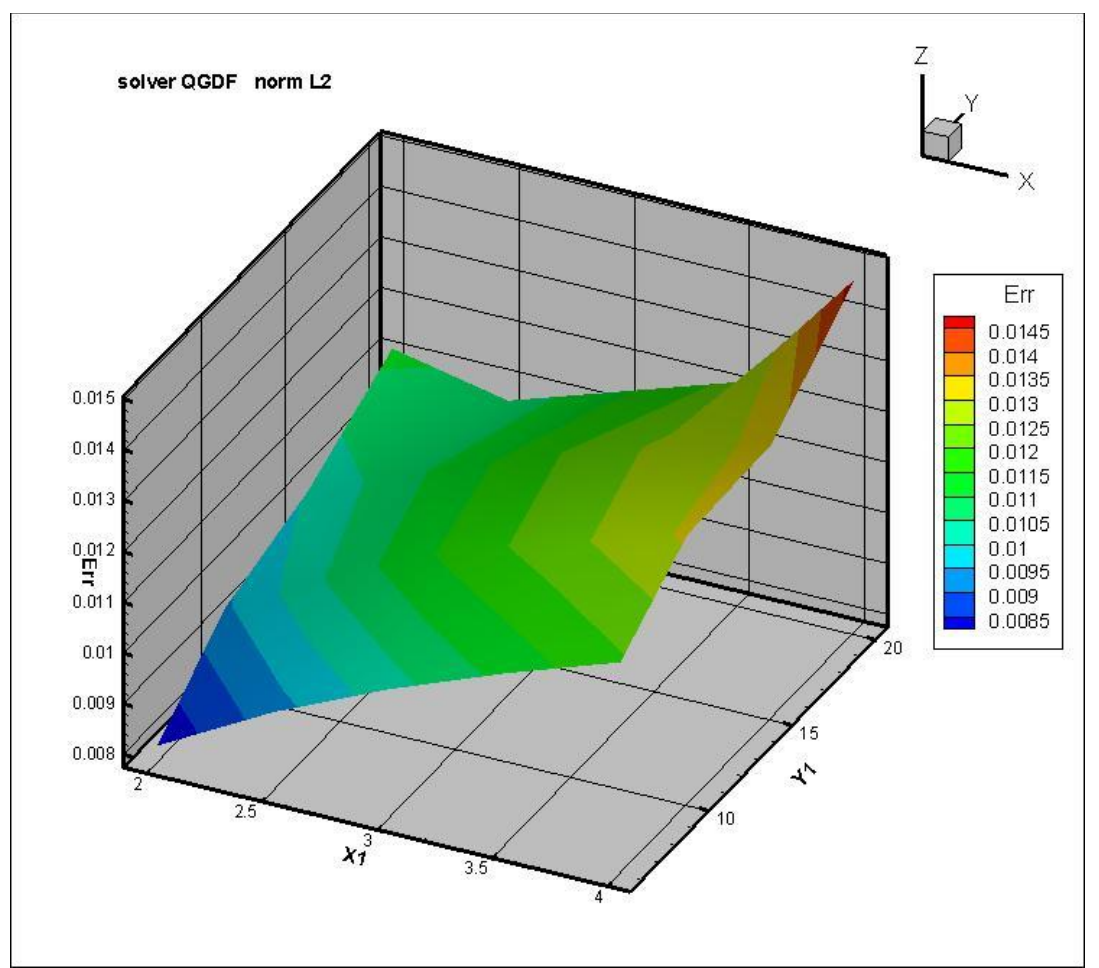

Fig. 7. The deviation from the exact solution in the L2 norm for the QGDFoam solver in the ranges of variation of the governing parameters

Now let's look at comparing all four solvers at different error norms. Figure 8 shows the deviations from the exact solution in the L1 norm for all solvers in the ranges of the governing parameters. Similarly, Figure 9 shows deviations from the exact solution in the L2 norm for all solvers.

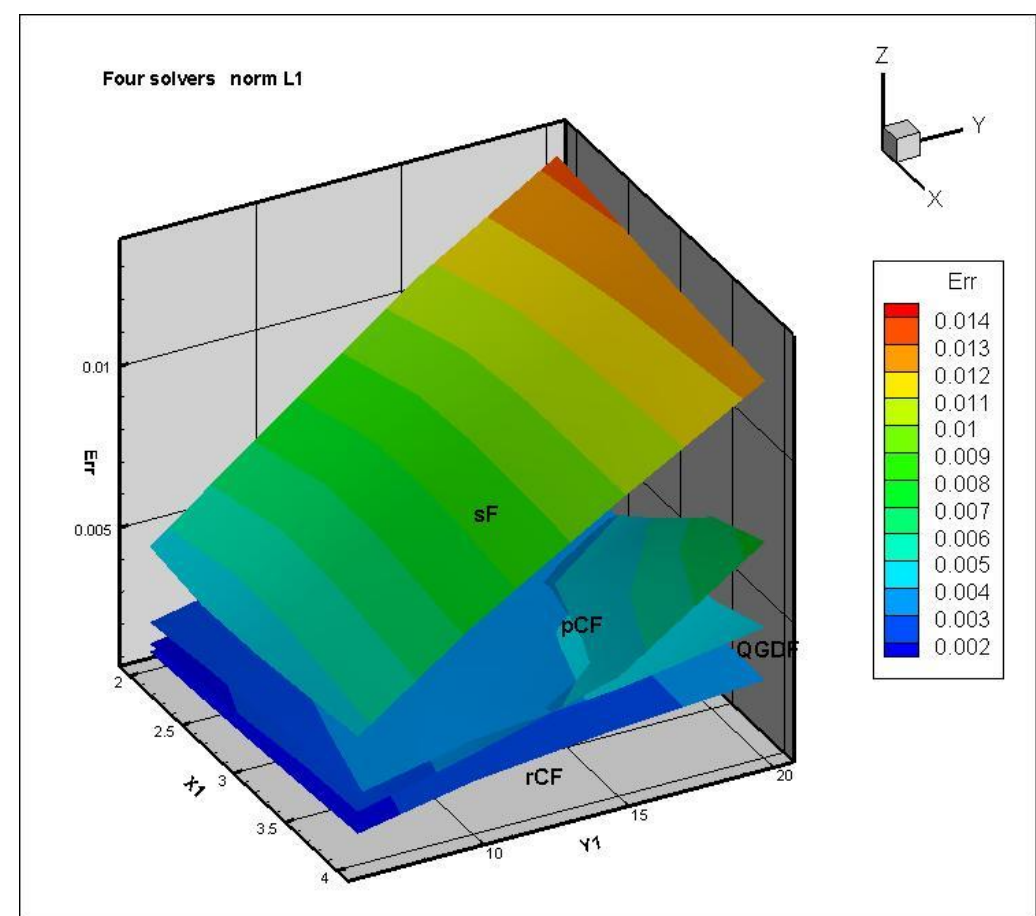

Fig. 8. The deviation from the exact solution in the L1 norm for all solvers in the ranges of the governing parameters. 


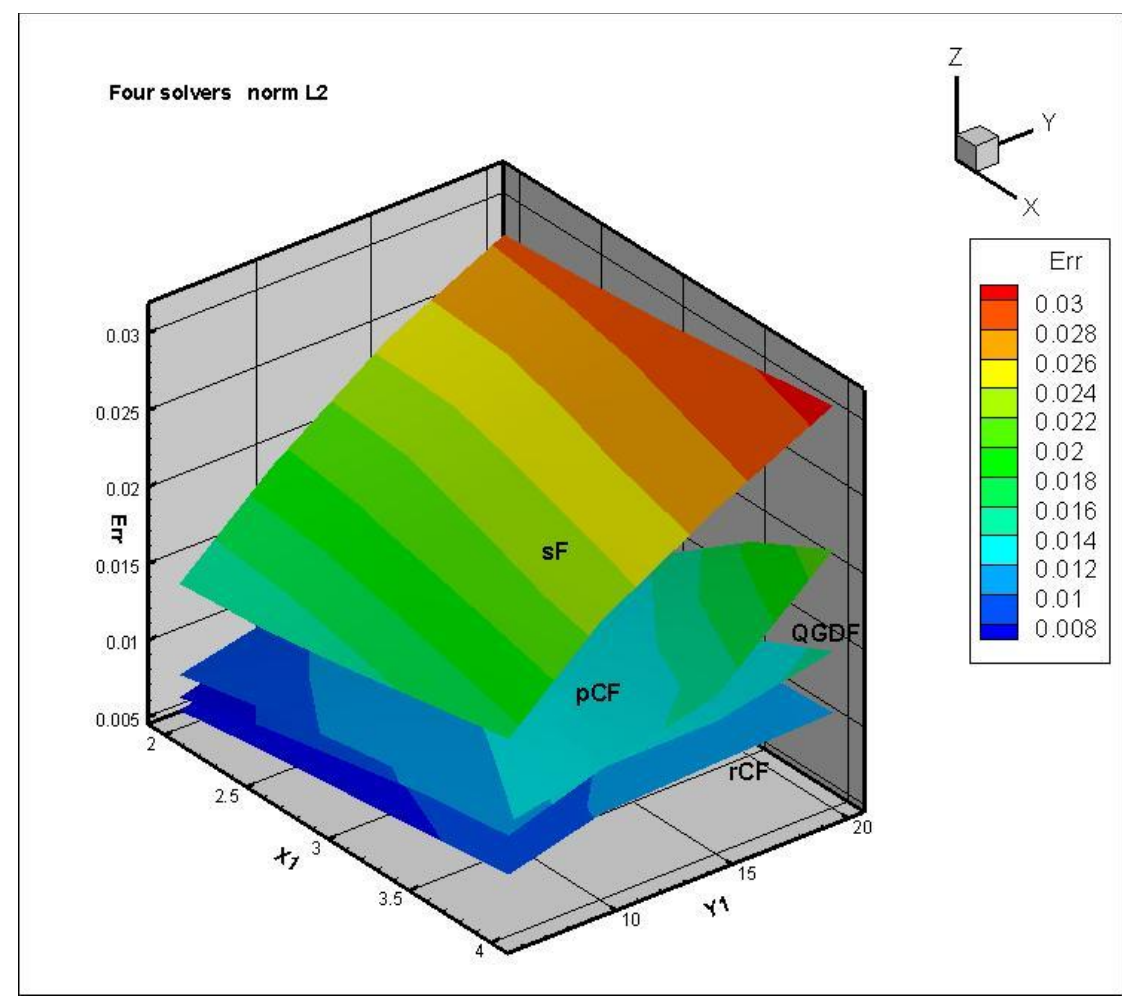

Fig. 9. The deviation from the exact solution in the L2 norm for all solvers in the ranges of the governing parameters.

As can be seen from Figures 8 and 9, qualitatively similar results were obtained for both norms. The rhoCentralFoam solver provides the smallest deviation from the exact solution. The next in order to deviate from the exact solution is the QGDFoam solver. The pisoCentralFoam solver gives slightly worse results. It should be noted that in the selected ranges of variation of the Mach number and the angle of deflection, the error surfaces for the QGDFoam and pisoCentralFoam solvers are located close to each other. The greatest deviation from the exact solution is observed in the results for SonicFoam solver.

Further, all the obtained surfaces can be presented in an analytical form according to the method proposed and described in [22].

According to [22], to approximate curved surfaces we will use second-order polynomials, where the error for the surface under consideration can be represented as a function of the following form:

$$
\mathrm{Err}=\mathrm{AX} 1+\mathrm{BY} 1+\mathrm{CX}_{1}^{2}+\mathrm{DY}_{1}^{2}+\mathrm{EX} 1 \mathrm{Y} 1+\mathrm{F}
$$

Here also $\mathrm{X} 1$ is the Mach number $\mathrm{M}, \mathrm{Y} 1$ is the flow deflection angle $\beta$, Err is the error of comparison with the exact solution in the L1 or L2 norm. Coefficients A, B, C, D. E, F are calculated for a specific surface.

Here is an example of a similar analytical dependence for a surface corresponding to the results for solver QGDFoam in the L2 norm (Fig. 9).

Approximating the required surface by a polynomial of the second order using the least squares method, we obtain the following values for the coefficients

$$
\begin{aligned}
& A=0.0007426759754917806 \\
& B=0.0005021159520976077 \\
& C=0.00020442857142857106 \\
& D=-0.000012584191705984598 \\
& E=-0.0000167566591422123 \\
& F=0.0038062569399619252
\end{aligned}
$$


For a more general comparative assessment, we will calculate the Error Index (EI), similarly to that proposed in [33]. According to [33], the Error Index (EI) is an average value for each error surface. The results for each solver in the L2 norm in accordance with Figure 9 are presented in Table 1.

Table 1. Error Index values for the problem of rarefaction wave formation

\begin{tabular}{|l|l|l|l|l|}
\hline Solver & rCF & QGDF & pCF & sF \\
\hline Error Index & 0.00894 & 0.01134 & 0.01182 & 0.02285 \\
\hline
\end{tabular}

Table 1 shows that the values of the error index EI fully correspond to the relative positions of the numerical results presented in Figure 9.

Thus, the results obtained in the form of visual representations of error surfaces, their analytical representations and calculated error indices allow the user of the above solvers to get a full understanding of their accuracy in the class of problems of a rarefaction wave formation.

\section{Conclusions}

The paper considers the processing and visualization of the results of a generalized computational experiment using a specific example. As an example, we used the results of a generalized computational experiment on the comparative assessment of the accuracy of four solvers of the OpenFOAM open software package. As a basic problem, the problem of the formation of a rarefaction wave when a supersonic flow of an inviscid gas flows around a plate at an angle of attack is used. The space of the defining parameters is set by the variation of two parameters in the selected ranges - the Mach number and the angle of flow deflection. For each solver, a discrete solution is obtained in the form of a dependence of the error on the defining parameters. A visual representation of the solution is shown, the example of analytical form of the solution is given as 2nd order polynomial.On the whole, the results obtained make it possible to estimate the degree of accuracy of each solver for the class of problems under consideration.

\section{Acknowledgements}

The study was supported by a grant from the Russian Science Foundation № 19-1100169, https://rscf.ru/project/19-11-00169/

\section{References}

[1] E. V. Myshenkov, V. I. Myshenkov, "Numerical simulation of efflux from Znamenskii's nozzle: Parametric investigations”, TVT, 37:1 (1999), 142-149; High Temperature, 37:1 (1999), 136-143

[2] K. N. Volkov, V. N. Emelyanov, M. S. Yakovchuk, "Multiparameter optimization of operating control by the trust vector based on the jet injection into the supersonic part of a nozzle", Num. Meth. Prog., 19:2 (2018), 158-172.

[3] Rahim M.F.A., Jaafar A.A., Mamat R., Taha Z. (2020) Parametric Study of CNG-DI Engine Operational Parameters by Using Analytical Vehicle Model. In: Osman Zahid M., Abd. Aziz R., Yusoff A., Mat Yahya N., Abdul Aziz F., Yazid Abu M. (eds) iMEC-APCOMS 2019. iMEC-APCOMS 2019. Lecture Notes in Mechanical Engineering. Springer, Singapore. https://doi.org/10.1007/978-981-15-0950-6 93

[4] Zangeneh, Rozie (2021) Parametric Study of Separation and Reattachment in Transonic Airfoil Flows, AIAA Journal, DOI: 10.2514/1.J060520

[5] Bondarev A.E. Analysis of Space-Time Flow Structures by Optimization and Visualization Methods // Transactions on Computational Science XIX, LNCS 7870, pp. 158-168.

[6] Bondarev A.E, Galaktionov V.A. Parametric Optimizing Analysis of Unsteady Structures and Visualization of Multidimensional Data // International Journal of Modeling, 
Simulation and Scientific Computing, 2013, V.04, N suppo1, 13 p., DOI 10.1142/S1793962313410043

[7] Bondarev A.E. Multidimensional data analysis in CFD problems / Scientific Visualization. V.6, № 5, p.59-66, 2014.

[8] Bondarev A.E., Galaktionov V.A. Multidimensional data analysis and visualization for time-dependent CFD problems // Programming and Computer Software, 2015, Vol. 41, No. 5, pp. 247-252, DOI:10.1134/So361768815050023

[9] Bondarev A.E. On the Construction of the Generalized Numerical Experiment in Fluid Dynamics // Mathematica Montisnigri, Vol. XLII, 2018, p. 52-64.

[10]A.E. Bondarev, V.A. Galaktionov. Generalized Computational Experiment and Visual Analysis of Multidimensional Data (2019). Scientific Visualization 11.4: 102 - 114, DOI: 10.26583/sv.11.4.09

[11]A.E. Bondarev . On visualization problems in a generalized computational experiment (2019). Scientific Visualization 11.2: 156 - 162, DOI: 10.26583/sv.11.2.12

[12] OpenFOAM Foundation. [Online]. Available: http://www.openfoam.org

[13] Loycyanskiy L.G. 1987 Mechanics of liquid and gas (Mehanika zhidkosti I gaza) (Moscow: Nauka) p 840 [In Russian]

[14] Abramovich G.N. 1976 Applied Gas Dynamics (Prikladnaya gazovaya dinamika) (Moscow: Nauka) p 888 [In Russian]

[15] Bondarev E.N., Dubasov V.T., Ryzhov Y.A., Svirschevsky S.B. and Semenchikov N.V. 1993 Aerigidromechanics (Aerogidromehanika). (Moscow: Mashinostroenie) p 608 [In Russian]

[16] Bondarev A.E., Galaktionov V.A., Chechetkin V. M. Analysis of the Development Concepts and Methods of Visual Data Representation in Computational Physics / Computational Mathematics and Mathematical Physics, 2011, Vol. 51, No. 4, pp. 624-636.

[17] Alexander E. Bondarev and Artem E. Kuvshinnikov. Analysis of the Accuracy of OpenFOAM Solvers for the Problem of Supersonic Flow Around a Cone // LNCS 10862, pp. 221-230, 2018. DOI:10.1007/978-3-319-93713-7_18

[18] Bondarev A., Kuvshinnikov A. Comparative Estimation of QGDFoam Solver Accuracy for Inviscid Flow Around a Cone // IEEE The Proceedings of the 2018 Ivannikov ISPRAS Open Conference (ISPRAS-2018). P. 82-87, DOI:10.1109/ISPRAS.2018.00019

[19] A.E. Bondarev, A.E. Kuvshinnikov. Analysis of the behavior of OpenFOAM solvers for 3D problem of supersonic flow around a cone at an angle of attack // CEUR Workshop Proceedings, V. 2763, 2020, p. 48-51. DOI: 10.30987/conferencearticle_5fce2771320efo.90086903

[20] Alekseev A.K., Bondarev A.E., Kuvshinnikov A.E. Comparative analysis of the accuracy of OpenFOAM solvers for the oblique shock wave problem // Matematica Montisnigri, 2019, vol. XLV, p. 95-105 DOI: 10.20948/mathmontis-2019-45-8

[21] Bondarev A., Kuvshinnikov A. Parametric Study of the Accuracy of OpenFOAM Solvers for the Oblique Shock Wave Problem // IEEE The Proceedings of the 2019 Ivannikov ISPRAS Open Conference (ISPRAS-2019) 2019. P. 108-112, DOI:10.1109/ISPRAS47671.2019.00023

[22] A.E. Bondarev. Processing of Visual Results of a Generalized Computational Experiment for the Problem of Supersonic Flow Around a Cone at an Angle of Attack (2021). Scientific Visualization 13.2: 104 - 116, DOI: 10.26583/sv.13.2.08

[23] C. Greenshields, H. Wellerr, L. Gasparini, J. Reese "Implementation of semi-discrete, non-staggered central schemes in a colocated, polyhedral, finite volume framework, for high-speed viscous flows", Int. J. Numer. Meth. Fluids, 63(1), 1-21 (2010). https://doi.org/10.1002/fld.2069

[24] A. Kurganov, E. Tadmor "New high-resolution central schemes for nonlinear conservation laws and convection-diffusion equations”, J. Comput. Phys., 160(1), 241-282 (2000). https://doi.org/10.1006/jcph.2000.6459 
[25] A. Kurganov, S. Noelle, G. Petrova, "Semidiscrete central-upwind schemes for hyperbolic conservation laws and Hamilton-Jacobi equations", SIAM J. on Sci. Comp., 23(3), 707740 (2001). https://doi.org/10.1137/S1064827500373413

[26] R. Issa, "Solution of the implicit discretized fluid flow equations by operator splitting", J. Comput. Phys., 62(1), 40-65 (1986). https://doi.org/10.1016/o021-9991(86)90099-9

[27] M. Kraposhin, A. Bovtrikova, S. Strijhak, "Adaptation of Kurganov-Tadmor numerical scheme for applying in combination with the PISO method in numerical simulation of flows in a wide range of Mach numbers", Procedia Computer Science, 66, 43-52 (2015). doi:10.1016/j.procs.2015.11.007.

[28] M. V. Kraposhin, D. A. Ryazanov, E. V. Smimova, T. G. Elizarova, M. A. Istomina, "Development of OpenFOAM Solver for Compressible Viscous Flows Simulation Using Quasi-Gas Dynamic Equations", Ivannikov ISPRAS Open Conference, 117-123 (2017).

[29] M. A. Istomina, "About realization of one-dimensional quasi-gas dynamic algorithm in the open program OpenFOAM complex", Preprint IPM No. 1 (Moscow: KIAM), (2018).

[30] B. N. Chetverushkin, T. N. Elizarova "Kinetic algorithms for calculating gas dynamic flows," U.S.S.R. Comput. Math. Math. Phys., 5 (5), 164-169 (1985).

[31] B. N. Chetverushkin, Kinetic schemes and Quasi-Gas Dynamic system of equations, CIMNE, Barcelona (2008).

[32] T. G. Elizarova, Quasi-Gas Dynamic Equations, Springer (2009).

[33] Aleksey Alekseev, Alexander Bondarev, Vladimir Galaktionov, Artem Kuvshinnikov, Lev Shapiro. On Applying of Generalized Computational Experiment to Numerical Methods Verification // CEUR Workshop Proceedings, V. 2744, 2020, p. paper19-1 - paper19-12, DOI: 10.51130/graphicon-2020-2-3-19 